\title{
A Hybrid Method for the Estimation of Power System Low-Frequency Oscillation Parameters
}

\author{
Jun-Zhe Yang, Member, IEEE, Chih-Wen Liu, Senior Member, IEEE, and Wen-Giang Wu
}

\begin{abstract}
A hybrid method which combines four different algorithms for low-frequency oscillation parameter estimation using wide-area measurements is presented in this paper. The first algorithm is the discrete Fourier transform which is used to start the low-frequency oscillation estimation process. The second one is the finite-impulse response (FIR) window filter which is employed in filtering unwanted components, and window correction factor is provided to eliminate the side effect of FIR window filter. The third one is the prototype algorithm which is proposed to estimate low-frequency oscillation parameters, and the last one is the least-square error method which is employed to enhance the computational accuracy by varying the window size. This hybrid method has been implemented in wide-area phasor measurements system by Java language and is under test in Taiwan's power system. Matlab/Simulink simulation and field test results are provided in the present paper.
\end{abstract}

Index Terms-Discrete Fourier transform, least-square error method, low-frequency oscillation, wide-area measurements.

\section{INTRODUCTION}

$\mathbf{P}$ OORLY damped low-frequency oscillation may cause a healthy power system into a stress condition under which the available transmission capability is reduced and even cascading outages may be initiated leading to a network collapse. Thus, estimation of low-frequency oscillation parameters for recognizing an evolving oscillation or for assessing the stability margin before a significant event occurs is essential for power system corrective and preventive control. Even post-oscillation estimation of parameters is important for analyzing the dynamic characteristics of studied power systems. Basically, there are two approaches to estimating the low-frequency oscillation parameters. They are dynamic model-based approach and measurement-based approach. The former compute the oscillation mode eigenvalue/eigenvector of dynamic system Jacobin matrix and can estimate the parameters in advance of an event, while the latter needs some sort of small oscillation measurement information to do the estimation. However, the dynamic model-based approach suffers from the inaccuracy of

Manuscript received November 21, 2006; revised June 9, 2007. This study was supported by a joint grant from the National Science Council, Taiwan, R.O.C., and ADX Company (NSC 94-2622-E-214-015-CC3). Paper no. TPWRS-00824-2006.

J.-Z. Yang is with the Department of Electrical Engineering, I-Shou University, Kaoshiung, Taiwan, R.O.C. (e-mail: jzyang@isu.edu.tw).

C.-W. Liu is with the Department of Electrical Engineering, National Taiwan University, Taipei, Taiwan, R.O.C. (e-mail: cwliu@cc.ee.ntu.edu.tw).

W.-G. Wu is with the ADX Company, Taiwan, R.O.C. (e-mail: a666@ms1. hinet.net)

Digital Object Identifier 10.1109/TPWRS.2007.907405 modeling process which is the common case, while the measurement-based approach is independent of this problem. Wide area measurement system consisting of phasor measurement units (PMUs) has been installed in many utilities [1]-[5] over the world to provide the opportunity to monitor low-frequency oscillations. Moreover, the data captured by a wide-area phasor measurements system (WAMS) requires techniques capable of extracting or estimating the parameters of interest.

In the past ten years, many algorithms have been applied to low-frequency oscillation parameter estimation using measurements, including the discrete Fourier transform (DFT) [6]-[9], the Kalman filter [10], the Prony method [11]-[16], and wavelet transform [17], and each has its own features. We try to give a brief comment on these methods, but it is far from a rigorous analysis. Easy implementation, efficient execution, and high noise-resistance are the main advantages of DFT, but the waveform of low-frequency oscillation is not a periodic sinusoidal wave. This makes DFT unable to directly estimate the parameters of low-frequency oscillation and without a compensating method. Kalman filter is a technique for estimating an unknown state of a dynamic system which has additive noise, but the assumed availability of signal dynamic model makes it inconvenient for low-frequency oscillation parameter estimation. Prony method is a very efficient tool and is able to estimate damping, amplitude, and frequency from measurement data. The Prony method has been successfully implemented for estimating power system dynamics [11], [12]. Lastly, wavelet transform is particularly appropriate in recognizing the features of signal in both the time and frequency domains, but wavelet analysis also has its own limitation: a correct wavelet is the key to correct analysis, unfortunately however, a damped sinusoid is not a wavelet.

The proposed hybrid method is composed of four parts, and each part has its own function. The first part is DFT which is used to start the low-frequency oscillation parameter estimation process. The second part is the finite impulse response (FIR) window filter which is employed to reject unwanted components and window correction factor (WCF) is provided to eliminate the side effect of FIR window filter. The third part is the prototype algorithm which we propose to estimate the low-frequency oscillation parameters, and the last part is the least-square error (LSE) method which is utilized to enhance the computational accuracy by varying the window size.

The organization of this paper is as follows. We describe each part of this hybrid method in Section II. Next, the test results are provided in Section III. Finally, we give the conclusions of the research in Section IV. 


\section{THE HYBRID METHOD}

\section{A. Starting Mechanism}

Since the proposed hybrid method uses the wide-area measurements to estimate the low-frequency oscillation parameters both on-line and off-line, it requires the starting mechanism to start the estimation in order to avoid invalid computing due to no enough dynamic information contained in measurements.

The variation in the phase angle of bus voltage phasor measurements is used to determine the start of the estimation since any small oscillation can cause changes in the phase angle. We start the process of low-frequency oscillation estimation in two types. The first type are the oscillations with larger amplitude in phase-angle difference. Usually, this type of oscillation is caused by serious disturbances. Therefore, the first type is started when the difference between the maximum angle and the minimum angle in $1 \mathrm{~s}$ is beyond the preset threshold. The second type is the oscillation with small amplitude. According to [6], [8], and [11], we chose Fourier analysis to start the second type of low-frequency oscillations parameter estimation, but we just do partial spectrum analysis. This is because oscillation frequency is known at about $0.8 \mathrm{~Hz}$ in the Taiwan power system (Taipower system). Meanwhile, the PMU uploads phasor data at a rate of $20 \mathrm{~Hz}$ in Taipower. Therefore, we use DFT with three different fundamental frequencies which are $0.5 \mathrm{~Hz}, 1 \mathrm{~Hz}$, and $2 \mathrm{~Hz}$, respectively, to compute the amplitudes of phase-angle difference in $1 \mathrm{~s}$. If one of these three amplitudes is beyond the threshold, small oscillation parameter estimation is started.

\section{B. Prototype Algorithm}

Since the FIR window filters we designed have a close relationship with the prototype algorithm we provided, we have to introduce the prototype algorithm before the FIR window filter.

When low-frequency oscillation occurs in a power system, we assume that the bus phase angle difference measurement, $x(k)$, can be expressed as follows:

$$
\begin{aligned}
x(k)=\sum_{i=1}^{p} X_{i} e^{\alpha_{i} k \Delta t} \cos \left(2 \pi f_{i} k \Delta t+\phi_{i}\right) & \\
& +X_{\mathrm{op}} e^{\beta k \Delta t}+X_{\mathrm{dc}}+\text { noise }
\end{aligned}
$$

where $p$ is the number of oscillation modes, $X_{i}$ is the amplitude, $\alpha_{i}$ is the decay constant, $f_{i}$ is the frequency, $\phi_{i}$ is the phase, $X_{\mathrm{dc}}$ is the dc component of the signal, $\Delta t$ is the time interval $(0.05$ $\mathrm{s})$, and $X_{\mathrm{op}} e^{\beta k \Delta t}$ is a decaying dc component which is used to represent the shift of the operating point. Since we will use the FIR window filter to filter $X_{\mathrm{dc}}$ and noise, we temporarily ignore these components in this section

$$
x(k)=\sum_{i=1}^{p} X_{i} e^{\alpha_{i} k \Delta t} \cos \left(2 \pi f_{i} k \Delta t+\phi_{i}\right)+X_{\mathrm{op}} e^{\beta k \Delta t}
$$

since we know that

$$
\cos \theta=\frac{e^{j \theta}+e^{-j \theta}}{2}
$$

then $x(k)$ can be expressed as

$$
\begin{array}{r}
x(k)=\frac{1}{2} \sum_{i=1}^{p} e^{\alpha_{i} k \Delta t}\left(X_{i} e^{-j \phi_{i}} e^{2 \pi f_{i} k \Delta t}+X_{i} e^{-j \phi_{i}} e^{-2 \pi f_{i} k \Delta t}\right) \\
+X_{\mathrm{op}} e^{\beta k \Delta t} \cdot
\end{array}
$$

For further derivations, we use the following definitions:

$$
\begin{aligned}
A_{i} & =\frac{X_{i}}{2} e^{j \phi_{i}} \\
a_{i} & =e^{j 2 \pi f_{i} \Delta t} \\
b_{i} & =e^{\alpha_{i} \Delta t} \\
c & =e^{\beta \Delta t} \\
z_{i} & =\operatorname{Re}\left(a_{i}\right) .
\end{aligned}
$$

Then (4) can be expressed as

$$
x(k)=\sum_{i=1}^{p} b_{i}^{k}\left(A_{i} a_{i}^{k}+\bar{A}_{i} a_{i}^{-k}\right)+X_{\mathrm{op}} c^{k} .
$$

Therefore, we can find the following relations between $x(k-1)$ and $x(k)$

$$
\begin{aligned}
x(k-1)= & \sum_{i=1}^{p} b_{i}^{k-1}\left(A_{i} a_{i}^{k-1}+\bar{A}_{i} a_{i}^{-(k-1)}\right)+\mathrm{X}_{\mathrm{op}} \mathrm{c}^{\mathrm{k}-1} \\
& \downarrow \times b_{i} \downarrow \times a_{i} \downarrow \times a_{i}^{-1} \downarrow \times c \\
x(k)= & \sum_{i=1}^{p} b_{i}^{k}\left(A_{i} a_{i}^{k}+\bar{A}_{i} a_{i}^{-k}\right)+\mathrm{X}_{\mathrm{op}} \mathrm{c}^{\mathrm{k}} .
\end{aligned}
$$

After some algebraic manipulations, we find

$$
\begin{aligned}
& \sum_{i=0}^{2 p+1} C_{i} x(k-i)=0 \\
& C=\left\{\{c,-1\} *\left\{b_{1},-2 z_{1}, b_{1}^{-1}\right\} *\left\{b_{2},-2 z_{2}, b_{2}^{-1}\right\} * \ldots \ldots\right. \\
& \left.\quad *\left\{b_{p-1},-2 z_{p-1}, b_{p-1}^{-1}\right\} *\left\{b_{p},-2 z_{p}, b_{p}^{-1}\right\}\right\}
\end{aligned}
$$

where $*$ represents the convolution operator. From (11) and (12), we have very useful consequences for follow-up development. We can use these two equations in two different ways. First, if the parameters of low-frequency oscillation are unknown, we can utilize (11) and the LSE method, which will be described in the last subsection to get the parameters. Second, if we already know the parameters of low-frequency oscillation, then we can apply (12) as a FIR window filter, which will be described in the next subsection.

Besides, from (12), it is obvious that the complexity of getting parameters is in direct proportion to the value of $\mathrm{p}$. Larger value of $p$ means more complicated algorithms and more computational time. Therefore, in the Taipower system, the value of $\mathrm{p}$ is chosen to be 1 for on-line application. As such, the waveform can be simplified as follows:

$x(k)=X_{1} e^{\alpha_{1} k \Delta t} \cos \left(2 \pi f_{1} k \Delta t+\phi_{1}\right)+X_{\mathrm{op}} e^{\beta k \Delta t}+X_{\mathrm{dc}}+$ noise. 


\section{FIR Window Filter}

Noise and some unwanted components are always in the signal. As these components will affect the accuracy of the computational results, a well-designed filter is essential to get rid of them.

Since digital filters are easy to design and implement, they have been widely used in signal processing. To get the precise low-frequency oscillation parameters, we have to design specific FIR window filters to filter not only noise but also dc component, decaying dc component, and decaying sinusoid component, respectively.

According to (12), the FIR window filters we designed are as follows:

$$
\begin{aligned}
& W_{a}=\left\{W_{F} *\{1,-1\}\right\} \\
& W_{b}=\{c,-1\} \\
& W_{c}=\left\{b_{1},-2 z_{1}, b_{1}^{-1}\right\}
\end{aligned}
$$

where $W_{a}$ is designed to filter noise and the dc component, $W_{b}$ is designed to filter the decaying dc component, and $W_{c}$ is designed to filter the decaying sinusoid component, and the chosen Blackman window is $W_{F}$.

By these FIR window filters, we can separate the decaying dc component and decaying sinusoid component, respectively from signal. Since there are two different decaying components in the signal and the decay constant of these two components should not be the same, one of them will fade faster than the other one. Therefore, to prevent the LSE method from encountering singular matrix or noise sensitivity, we estimate the parameters of these components separately.

The steps we take to separately estimate the parameters of low-frequency oscillation are as follows.

Step 1) When low-frequency oscillation has been detected, the signal $x(k)$ will be filtered by $W_{a}$ to get rid of noise and dc component and to get $\widetilde{x}_{1}(k)$. Therefore, the main components in the $\widetilde{x}_{1}(k)$ are the decaying dc component and the decaying sinusoid component.

Step 2) The initial value of $c$ is 1 .

Step 3) $\widetilde{x}_{1}(k)$ is filtered by $W_{b}$ to get rid of the decaying dc component and to get $\widetilde{x}_{2}(k)$. Therefore, the main component in the $\widetilde{x}_{2}(k)$ is the decaying sinusoid component.

Step 4) By utilizing (11) and the LSE method, the parameters, $b_{1}$ and $z_{1}$ can be estimated.

Step 5) $\widetilde{x}_{1}(k)$ is filtered by $W_{c}$ to get rid of the decaying sinusoid component and to get $\widetilde{x}_{3}(k)$. Therefore, the main component in the $\widetilde{x}_{3}(k)$ is the decaying dc component.

Step 6) By utilizing (11) and the LSE method, the parameter $c$, can be estimated.

According to the experiments, after repeating Steps 3 to 6 three times, the values of $b_{1}, z_{1}$, and $c$ will be converged, where $\widetilde{x}_{1}(k)$, $\widetilde{x}_{2}(k), \widetilde{x}_{3}(k)$ can be obtained as follows:

$$
\widetilde{x}_{1}(k)=\sum_{m=0}^{M-1} W_{a m} x(k-m)
$$

$$
\begin{aligned}
& \widetilde{x}_{2}(k)=\sum_{m=0}^{1} W_{b m} \widetilde{x}_{1}(k-m) \\
& \widetilde{x}_{3}(k)=\sum_{m=0}^{2} W_{c m} \widetilde{x}_{1}(k-m)
\end{aligned}
$$

where $M$ is the window size of $W_{a}$.

Then, according to the definitions (6)-(9) given above, we can estimate the parameters of low-frequency oscillation by means of the following equations:

$$
\begin{aligned}
f_{1} & =\frac{\cos ^{-1}\left(z_{1}\right)}{2 \pi \Delta t} \\
\alpha_{1} & =\frac{\ln \left(b_{1}\right)}{\Delta t} \\
\beta & =\frac{\ln (c)}{\Delta t .}
\end{aligned}
$$

However, other problems encountered are phase shift and amplitude modulation, which are the side effects of using FIR window filter. To eliminate these problems, the Window Correction Factor (WCF) is presented.

From (13), the signal $x(k)$ is filtered by $W_{a}$ to get $\widetilde{x}_{1}(k)$ in (17), and then $\widetilde{x}_{1}(k)$ is filtered by $W_{b}$ to get $\widetilde{x}_{2}(k)$ in (18). Therefore, $\widetilde{x}_{2}(k)$ can be expressed as

$\widetilde{x}_{2}(k)=A_{1} a_{1}^{k} b_{1}^{k} \sum_{m=0}^{M} a_{1}^{m} b_{1}^{m} W_{d m}+\bar{A}_{1} a_{1}^{-k} b_{1}^{k} \sum_{m=0}^{M} a_{1}^{-m} b_{1}^{m} W_{d m}$

where

$$
W_{d}=\left\{W_{F} *\{1,-1\} *\{c,-1\}\right\}
$$

According to (5), (10), and (23), we obtain

$$
\begin{aligned}
A_{1} & =\frac{a_{1} b_{1}^{-1} \widetilde{x}_{2}(k+1)-\widetilde{x}_{2}(k)}{\left(a_{1}^{2}-1\right) \times \mathrm{WCF}_{1}} \\
X_{1} & =2\left|A_{1}\right| \\
\phi_{1} & =\operatorname{angle}\left(A_{1}\right)
\end{aligned}
$$

where $\mathrm{WCF}_{1}$ is defined as follows:

$$
\mathrm{WCF}_{1}=\sum_{m=0}^{M} a_{1}^{m} b_{1}^{m} W_{d m}
$$

By WCF, the side effects of using FIR window filter can be totally eliminated, and the amplitude and phase angle of lowfrequency oscillation can be estimated precisely.

\section{LSE Method}

We utilize (11) and the LSE method to estimate the parameters of low-frequency oscillation. This is because the LSE method is easy to implement and the data window size of the LSE method can be adjusted according to the frequency of low-frequency oscillation. Since the frequency range of low-frequency oscillations is between 0.2 to $3 \mathrm{~Hz}$, it is certain that a larger window size is needed for obtaining more accurate estimation when the frequency is lower than $1 \mathrm{~Hz}$. Therefore, 
from (18), and using the consequences of (11) and (12), we obtain

$$
-b_{1} \widetilde{x}_{2}(k)+2 z_{1} \widetilde{x}_{2}(k+1)-b_{1}^{-1} \widetilde{x}_{2}(k+2)=0 .
$$

Rearrange (29) as

$$
\left[\begin{array}{cc}
\widetilde{x}_{2}(k) & \widetilde{x}_{2}(k+1) \\
\widetilde{x}_{2}(k+1) & \widetilde{x}_{2}(k+2) \\
\vdots & \vdots \\
\widetilde{x}_{2}(k+L-3) & \widetilde{x}_{2}(k+L-2)
\end{array}\right]\left[\begin{array}{l}
p_{1} \\
p_{2}
\end{array}\right]=\left[\begin{array}{c}
\widetilde{x}_{2}(k+2) \\
\widetilde{x}_{2}(k+3) \\
\vdots \\
\widetilde{x}_{2}(k+L-1)
\end{array}\right]
$$

where $L$ is the data window size, and

$$
\begin{aligned}
& p_{1}=-b_{1}^{2} \\
& p_{2}=2 z_{1} b_{1} .
\end{aligned}
$$

Here, we can simplify (30) to the following:

$$
\mathbf{A P}=\mathbf{B} .
$$

By the LSE method, $\mathbf{P}$ can be obtained as

$$
\mathbf{P}=\left(\mathbf{A}^{\mathrm{T}} \mathbf{A}\right)^{-1} \mathbf{A}^{\mathrm{T}} \mathbf{B} .
$$

By (31) and (32), we can obtain

$$
\begin{aligned}
b_{1} & =\sqrt{-p_{1}} \\
z_{1} & =\frac{p_{2}}{2 b_{1} .} .
\end{aligned}
$$

We also use the LSE method to estimate the parameter $c$. After getting $b_{1}$ and $z_{1}$, we utilize (16), (19), and the consequences of (11) and (12), and obtain

$$
\left[\begin{array}{c}
\widetilde{x}_{3}(k) \\
\widetilde{x}_{3}(k+1) \\
\vdots \\
\widetilde{x}_{3}(k+L-3)
\end{array}\right][c]=\left[\begin{array}{c}
\widetilde{x}_{3}(k+1) \\
\widetilde{x}_{3}(k+2) \\
\vdots \\
\widetilde{x}_{3}(k+L-2)
\end{array}\right]
$$

By the LSE method, we obtain

$$
c=\frac{\sum_{i=0}^{L-3} \widetilde{x}_{3}(k+i) \widetilde{x}_{3}(k+i+1)}{\sum_{i=0}^{L-3} \widetilde{x}_{3}^{2}(k+i)} .
$$

Now, these four subsections can be summarized as illustrated in the flowchart in Fig. 1. In Fig. 1, the window size of $W_{a}$ is 19. Moreover, the window size of LSE method is changing to meet the need of both quick response and accurate estimation. The smallest window size of LSE method we set is 5 and the largest window size of LSE method we set is $40(L=5 \sim 40)$.

\section{Test Results}

In this section, we present some simulation and field test results to demonstrate the performance of this hybrid method. The
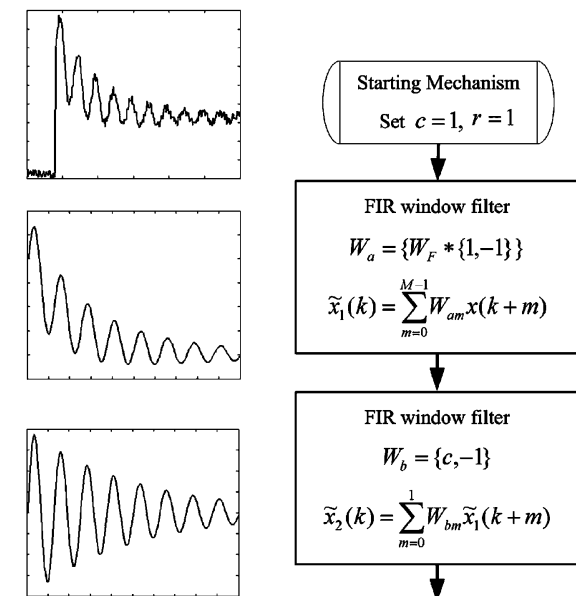

FIR window filter

$W_{a}=\left\{W_{F} *\{1,-1\}\right\}$

$\widetilde{x}_{1}(k)=\sum_{m=0}^{M-1} W_{a m} x(k+m)$

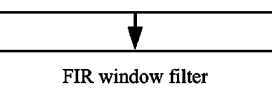

$W_{b}=\{c,-1\}$

$\tilde{x}_{2}(k)=\sum_{m=0}^{1} W_{b m} \widetilde{x}_{1}(k+m)$
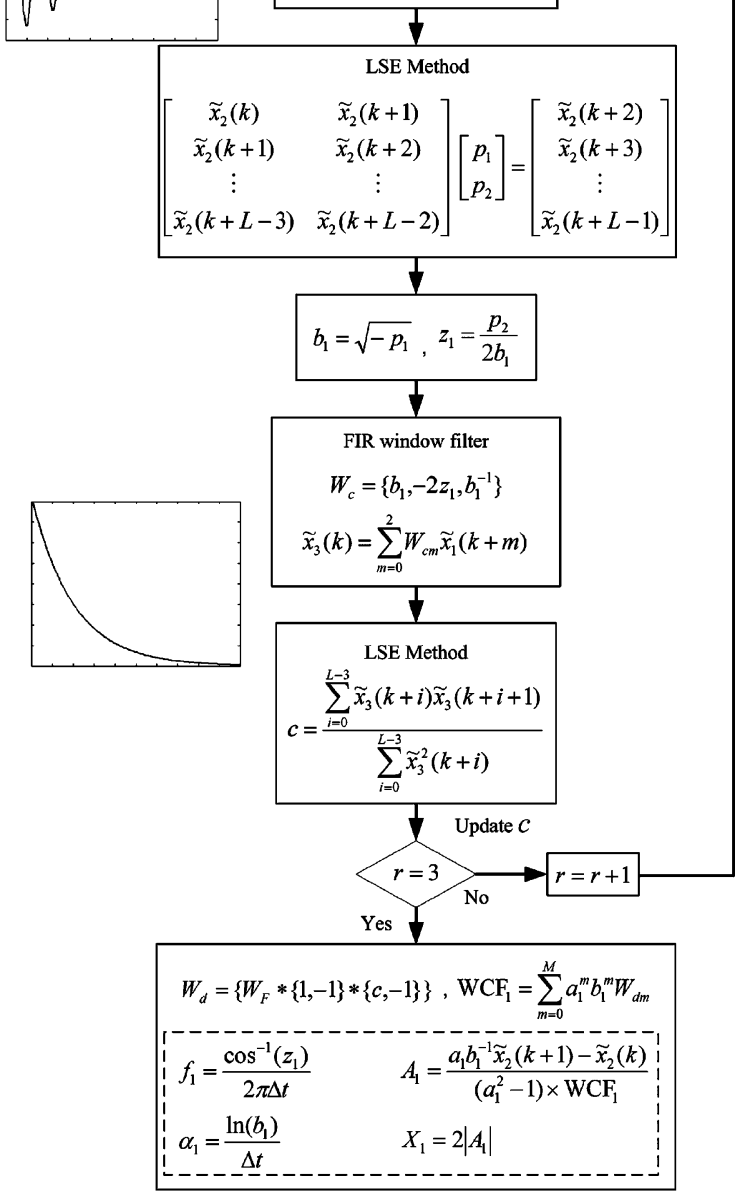

Fig. 1. Computation flowchart.

simulation tests are performed by Matlab/Simulink, and the field measurement data are provided by the ADX Company.

\section{A. Matlab/Simulink Simulation Tests}

To demonstrate the online performance of this hybrid method, we simulate a two-machine transmission system by Matlab/Simulink, as shown in Fig. 2. Two simulated ADX-3000s, which are PMUs produced by ADX Company are installed in Bus 1 (B1) and Bus 2 (B2), respectively, and the 


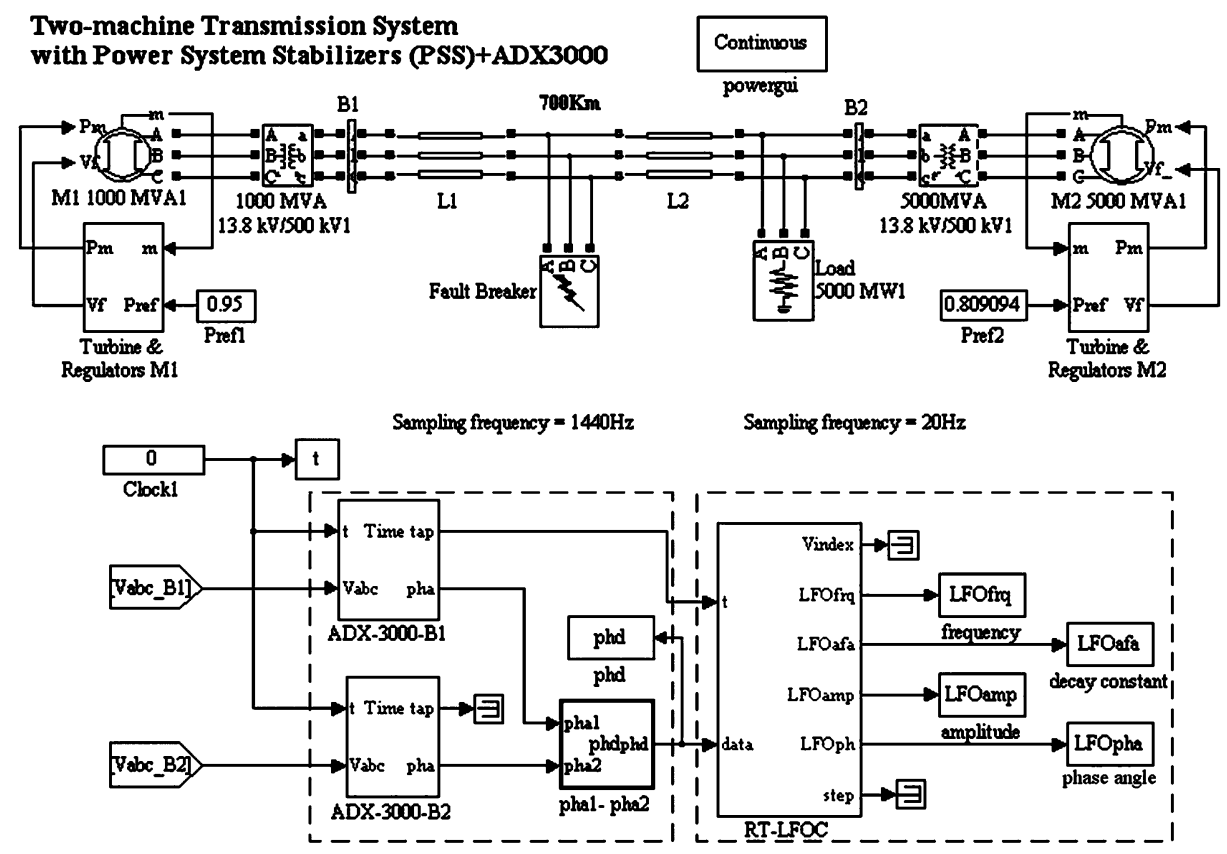

Fig. 2. Simulation structure for real-time low-frequency oscillation estimation.

TABLE I

PARAMETERS USED IN THE SIMULATION SYSTEM

\begin{tabular}{|c|c|c|c|c|c|}
\hline Generator MI : & & Generator M2 : & & Transformer: & \\
\hline $\mathrm{Pn}$ & 1000MVA & Pn & 5000MVA & $\mathrm{V} 1$ & $13.8 \mathrm{KV}$ \\
\hline$V_{n}$ & $13.8 \mathrm{KV}$ & $V_{n}$ & $13.8 \mathrm{KV}$ & V2 & $500 \mathrm{KV}$ \\
\hline Fn & $60 \mathrm{~Hz}$ & Fn & $60 \mathrm{~Hz}$ & R1 & $0.002 \mathrm{pu}$ \\
\hline $\mathrm{Xd}$ & 1.305 & $\mathrm{Xd}$ & 1.305 & Ll & $0 \mathrm{pu}$ \\
\hline $\mathrm{Xd}$ & 0.296 & $\mathrm{Xd}$ & 0.296 & $\mathrm{R} 2$ & $0.002 \mathrm{pu}$ \\
\hline $\mathrm{Xd"}$ & 0.252 & $\mathrm{Xd"}$ & 0.252 & $\mathrm{~L} 2$ & 0.12 pu. \\
\hline $\mathrm{Xq}$ & 0.474 & $\mathrm{Xq}$ & 0.474 & & \\
\hline $\mathrm{Xq}^{\prime \prime}$ & 0.243 & $\mathrm{Xq}^{\prime \prime}$ & 0.243 & Line: & (ohms $/ \mathrm{km}$ ) \\
\hline $\mathrm{XI}$ & 0.18 & XI & 0.18 & $\mathrm{R} !$ & 0.01755 \\
\hline $\mathrm{Td}^{\prime}$ & 1.01 & Td' & 1,01 & R0 & 0.2758 \\
\hline $\mathrm{Td}^{\prime \prime}$ & 0.053 & $\mathrm{Td}^{\prime \prime}$ & 0.053 & $\mathrm{LI}$ & $8.74 \mathrm{E}-04$ \\
\hline Tqo" & 0.1 & Tqo" & 0.1 & Lo & $3.22 \mathrm{E}-03$ \\
\hline Rs & $2.85 \mathrm{E}-03$ & Rs & $2.85 \mathrm{E}-03$ & $\mathrm{Cl}$ & 1.33E-08 \\
\hline H & 3.7 & $\mathrm{H}$ & 3.7 & $\mathrm{CO}$ & $8.30 \mathrm{E}-09$ \\
\hline
\end{tabular}

length of the transmission line is $700 \mathrm{~km}$. The hybrid method is performed by Embedded Matlab Function, and the input data are the phase angle difference between B1 and B2. A single-phase ground fault occurs at $0.1 \mathrm{~s}$ and is cleared at 0.2 $\mathrm{s}$, and the total simulation time is $9 \mathrm{~s}$. The parameters used in Fig. 2 are provided in Table I. Two different cases are provided in this paper. One is the unstable case, and the other is the stable case.

Case 1: These two machines turn off the power system stabilizer (PSS), and the fault occurs at the middle of the transmission line. The solid line in Fig. 3(a) shows the phase angle difference between B1 and B2, and Fig. 3(b), (c), and (d) show the frequency, decay constant, and amplitude of oscillation, respectively. From Fig. 3(a) and (3d), the phase angle difference between B1 and B2 increases rapidly after the fault occurs. Fig. 3(b) and Fig. 3(c) shows an oscillation frequency of about $0.9 \mathrm{~Hz}$ and positive decay constant, respectively. From these figures, it is clear that the system is unstable.
Case 2: Machine M1 turns on the PSS, but M2 still turns off the PSS, and the fault occurs at $550 \mathrm{~km}$ from the distance of B1. The dotted line in Fig. 3(a) shows the phase angle difference between B1 and B2, and Fig. 3(b), (c), and (d) show the frequency, decay constant, and amplitude of oscillation, respectively. In this case, the system is stable, because the decay constant is negative. Moreover, the computation stopped when the amplitude of oscillation is less than the threshold.

From these two cases, we can find that the hybrid method can be applied for online application and the decay constant is a good index to show the tendency of the system. Therefore, the damping ratio, as shown in (39), is a very important index in field application

$$
\text { damping ratio }=\frac{-\alpha_{1}}{\sqrt{\alpha_{1}^{2}+\left(2 \pi f_{1}\right)^{2}}} \times 100 \% .
$$

\section{B. Field Data Tests}

Low-frequency oscillation phenomena have been observed in the Taipower system from 1984. To prevent the damage caused by low-frequency oscillation, several generators in the Taipower system have been installed with power system stabilizers (PSSs). However, with the increase of load and shortage of transmission capacity, low-frequency oscillation is still a threat to Taipower system. More than ten PMUs, which were produced by the local ADX Company, have been installed into the backbone of Taipower system, and each PMU uploads its time-tagged phasor data at a rate of $20 \mathrm{~Hz}$ to the central servers in Taipower building and Taipower Company Power Research Institute. This quantity of information is enough for Taipower Company to monitor the dynamic state of the Taipower system or to develop more applications. Low-frequency oscillation parameters estimation is one of these applications. 


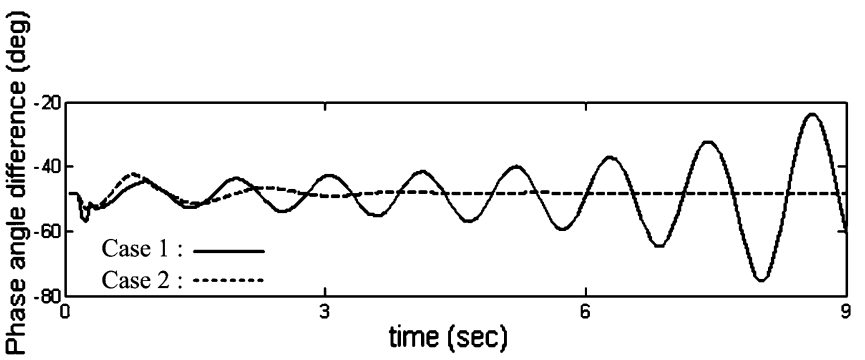

(a)

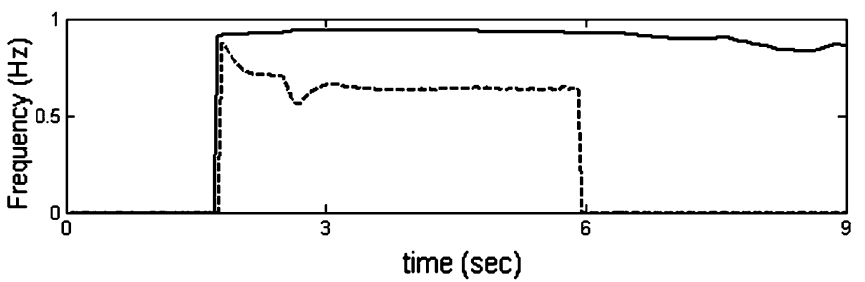

(b)

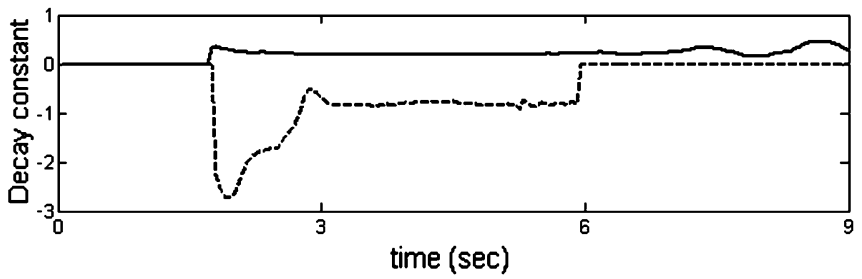

(c)

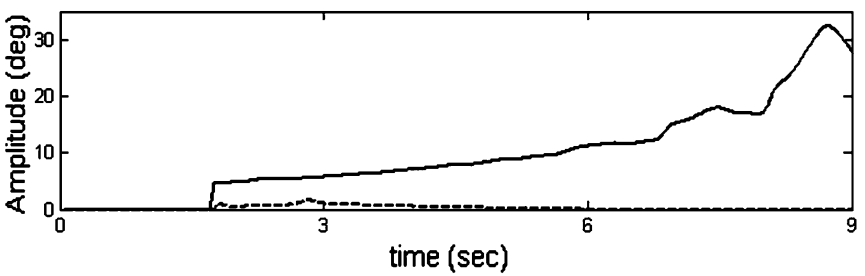

(d)

Fig. 3. Simulation and computation results (on-line version).

The measured data of PMUs in the Taipower system can be categorized into two types: the real-time data which is updated at a rate of $20 \mathrm{~Hz}$ and historical data which are contained in the database. Hence, to meet the on-line and off-line applications and requirements, we have developed two different versions of this hybrid method. Furthermore, these two versions are implemented by Java language and are under test in Taipower system.

The off-line version provides easy-reading information for the administrative department. It gives data such as the time when oscillation actually started, the principal frequency and damping ratio, and the duration of the oscillation. Since the offline version uses historical data for estimation, it can estimate the parameters when oscillation occurs.

Moreover, the on-line version is provided to help central operators of Taipower Company understand the real dynamic state. Since the on-line version uses real-time data for estimation, it is restricted by the data rate and estimates the parameters in a fixed window.

Fig. 4 shows the map of Taipower system. Taiwan is a long, narrow, and mountainous island. Since most of its mountains are

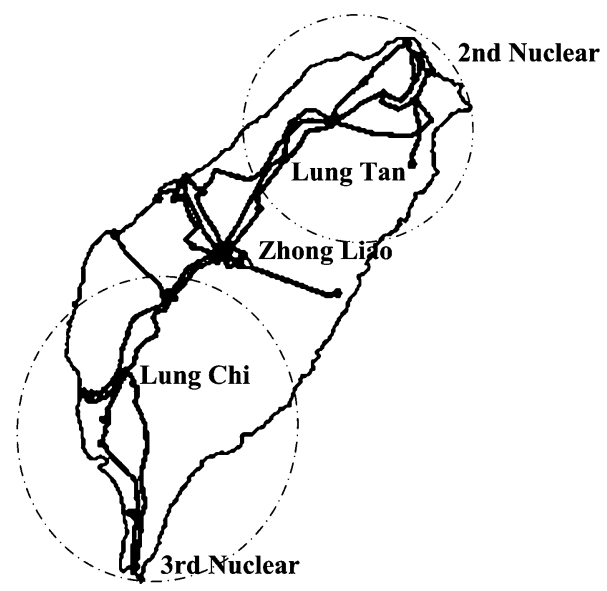

Fig. 4. Diagram of Taipower system.

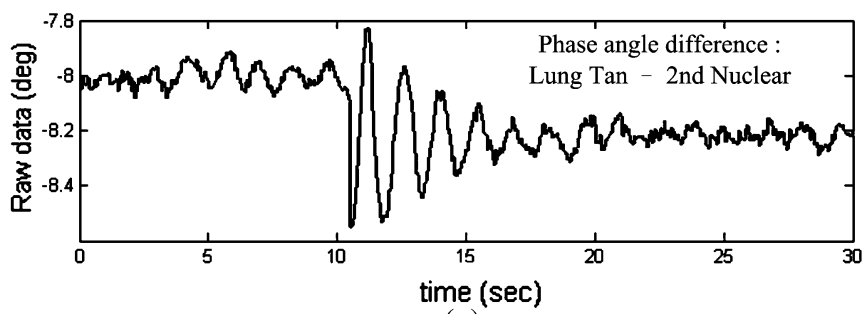

(a)

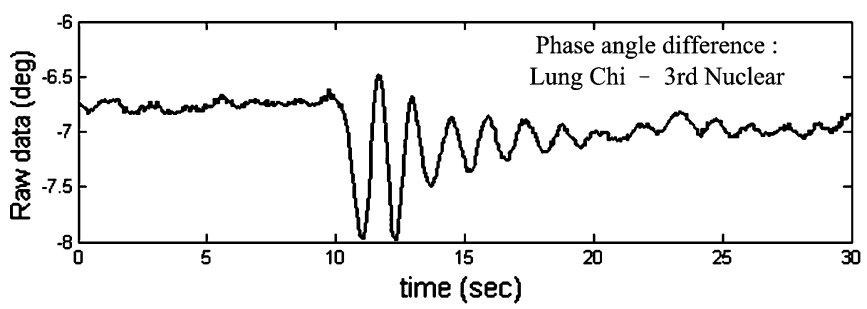

(b)

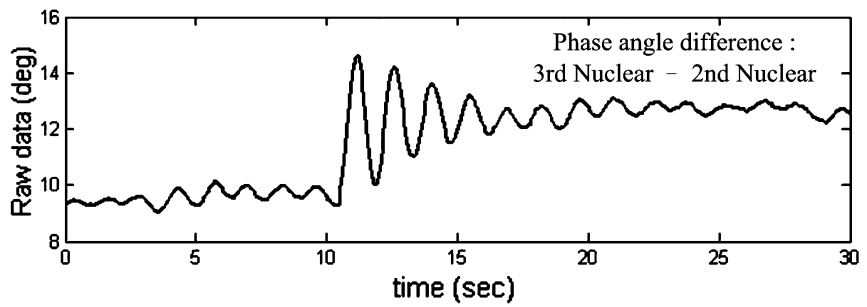

(c)

Fig. 5. Field data.

in the east, the major development area is in the west. Accordingly, the major electric transmission lines are almost located in the western Taiwan as shown in the bold solid lines on the map. Since the major loads are located in northern Taiwan and most generating plants are in the middle and southern Taiwan, significant power had been transferred from south to north. Therefore, inter-area mode oscillations are the major concerns of Taipower system.

On January 24, 2004, some faults happened in the Taipower system, which caused a mild oscillation in the Taipower system. The field data is shown in Fig. 5 and computational results are shown in Fig. 6. 


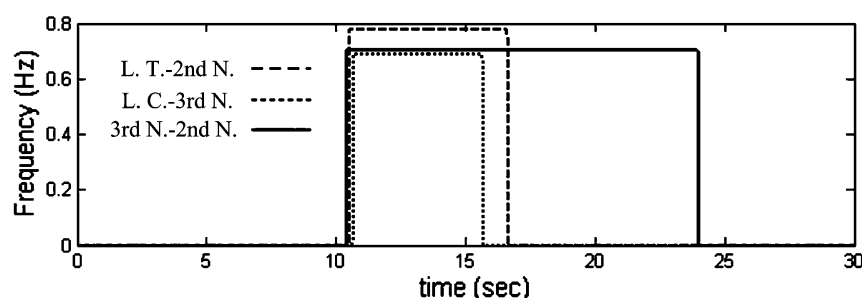

(a)

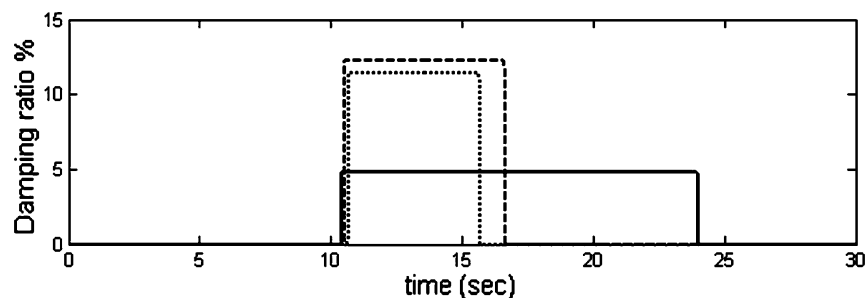

(b)

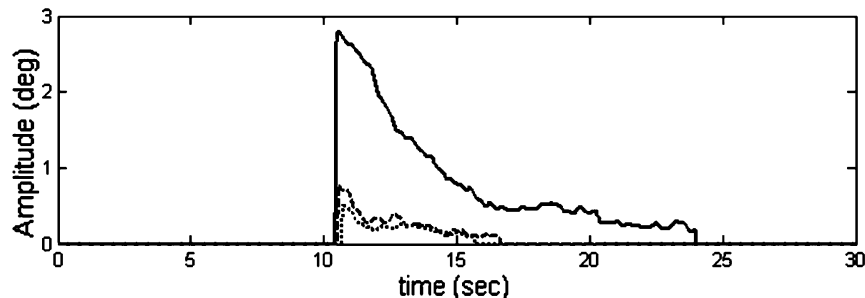

(c)

Fig. 6. Computational results (off-line version)

Fig. 5(a) shows the phase angle difference between Lung Tan and 2nd Nuclear which are in the northern Taiwan and Fig. 5(b) shows the phase angle difference between Lung Chi and 3rd Nuclear, which are in the southern Taiwan. It is clear that the oscillation in northern and southern Taipower system is smaller than the oscillation between 3rd Nuclear and 2nd Nuclear as shown in Fig. 5(c). This means that Taipower Company still has to keep an eye on inter-area mode oscillations.

Fig. 6(a) shows the frequencies of oscillation, which are all lower than $0.8 \mathrm{~Hz}$. Fig. 6(b) shows the damping ratios of oscillation, and the damping ratio of inter-area mode is worse than that of local mode. Moreover, Fig. 6(c) shows the amplitudes of oscillation, and it is obvious that the amplitude of inter-area mode oscillation is bigger then the others.

\section{Multi-Mode Tests}

For further analysis and application, we demonstrate this hybrid method can be used in two multi-mode examples.

From the flowchart in Fig. 1, it is easy to understand that this hybrid method estimates the parameters of each component in the signal by separating them with FIR window filters. It is the same for multi-mode cases. Although this way takes more computational time on iteration, it makes sure this hybrid method would not suffer singular matrix when some components fade away.

The first multimode example is shown in Table II. A simulated signal $x(k)$ containing three different decaying sinusoid components is provided to be the input data, and the data length is 50 . We change the frequencies of each component to observe
TABLE II

Computational Results of Different FreQuencies

\begin{tabular}{|c|c|c|c|}
\hline \multicolumn{4}{|c|}{$f_{1}=0.5, f_{2}=0.8, f_{3}=1.1$} \\
\hline Hybrid Method & frequency & decay constant & amplitude \\
\hline$f_{1}$ & 0.5000 & -0.1000 & 1.0000 \\
\hline$f_{2}$ & 0.8000 & -0.4000 & 4.0000 \\
\hline$f_{3}$ & 1.1000 & -0.2000 & 0.5000 \\
\hline
\end{tabular}

(a)

\begin{tabular}{|c|c|c|c|}
\hline \multicolumn{4}{|c|}{$f_{1}=0.6, f_{2}=0.8, f_{3}=1.0$} \\
\hline Hybrid Method & frequency & decay constant & amplitude \\
\hline$f_{1}$ & 0.6000 & -0.0999 & 0.9997 \\
\hline$f_{2}$ & 0.8000 & -0.4000 & 4.0001 \\
\hline$f_{3}$ & 1.0000 & -0.2001 & 0.5002 \\
\hline
\end{tabular}

(b)

\begin{tabular}{|c|c|c|c|}
\hline \multicolumn{4}{|c|}{$f_{1}=0.7, f_{2}=0.8, f_{3}=0.9$} \\
\hline Hybrid Method & frequency & decay constant & amplitude \\
\hline$f_{1}$ & 0.7049 & -0.0352 & 0.9469 \\
\hline$f_{2}$ & 0.8025 & -0.3399 & 4.2909 \\
\hline$f_{3}$ & 0.9023 & -0.0715 & 0.3556 \\
\hline
\end{tabular}

(c)

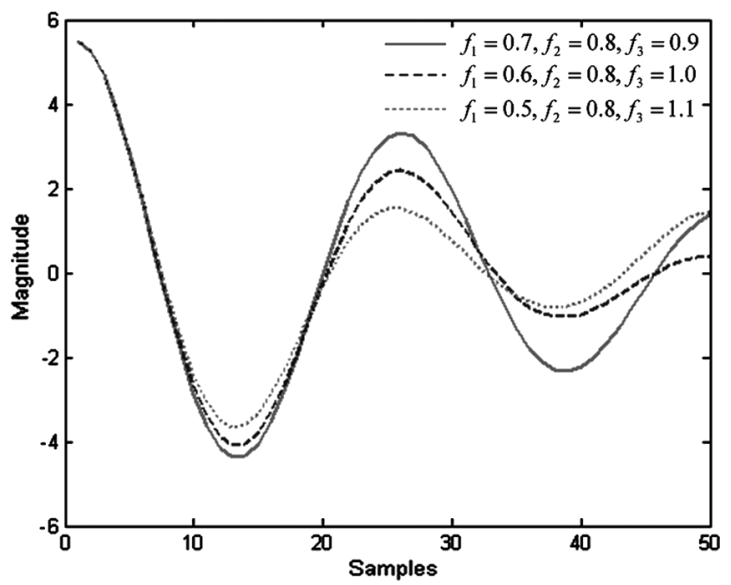

Fig. 7. Simulated signals.

the discrimination performance as shown in Fig. 7. It is clear from Tables II(a), (b), and (c) that as the frequencies of each component close, the computational results become worse. However, the discrimination performance can be restrictedly improved by increasing the number of iteration or the data length.

In the second multimode example, we extract the input data (as shown in Fig. 8) from the field data provided in Fig. 5(a). After the input data is filtered by $W_{a}$ to eliminate noise and dc component, the filtered data is computed by the hybrid method. Since we do not exactly know the number of oscillation modes in the filed signal, we estimate the parameters by assuming $\mathrm{p}=$ $1, p=2$ and $p=3$ to evaluate the affect of $p$ value on parameters estimation. Table III shows that the computational results of different number of oscillation modes, and one can see the 


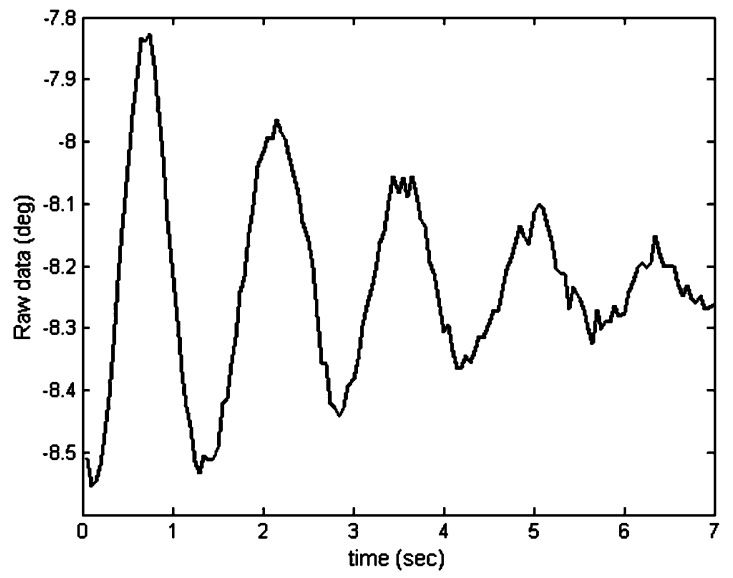

Fig. 8. Test data for hybrid method.

TABLE III

COMPUTATIONAL RESUltS FOR $\mathrm{p}=1, \mathrm{p}=2$ AND $\mathrm{p}=3$

\begin{tabular}{|c|c|c|c|}
\hline \multicolumn{4}{|c|}{$p=1$} \\
\hline Hybrid Method & frequency & decay constant & amplitude \\
\hline$f_{1}$ & 0.7345 & -0.3249 & 0.4555 \\
\hline
\end{tabular}

(a)

\begin{tabular}{|c|c|c|c|}
\hline \multicolumn{4}{|c|}{$p=2$} \\
\hline Hybrid Method & frequency & decay constant & amplitude \\
\hline$f_{1}$ & 0.6990 & -0.3153 & 0.4547 \\
\hline$f_{2}$ & 1.4124 & -1.0925 & 0.1268 \\
\hline
\end{tabular}

(b)

\begin{tabular}{|c|c|c|c|}
\hline \multicolumn{4}{|c|}{$p=3$} \\
\hline Hybrid Method & frequency & decay constant & amplitude \\
\hline$f_{1}$ & 0.6982 & -0.3461 & 0.4858 \\
\hline$f_{2}$ & 1.4470 & -1.1615 & 0.1248 \\
\hline$f_{3}$ & 3.7617 & -27.0575 & 0.1234 \\
\hline
\end{tabular}

(c)

estimated parameters of oscillation are insensitive to assumed $p$ value. This is a remarkable feature of the proposed method.

\section{Very Small Oscillation Tests}

By reducing the threshold of the starting mechanism, this hybrid method can be used in very small oscillations as shown in Fig. 9. The raw data in Fig. 9(a) is phase angle difference between Lung Chi and Lung Tang at 12:24 on July 13, 2006. Fig. 9(b), (c), and (d) shows the computational results of frequency, damping ratio and amplitude. From these results, the proposed hybrid method can indeed estimate low-frequency oscillation parameters for both small and large oscillations. This feature makes the proposed method can be used as a warning signal for operators to take necessary preventive actions before a significant event indeed occurs.

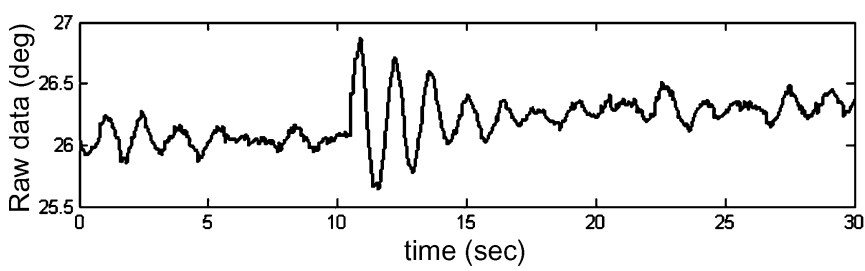

(a)

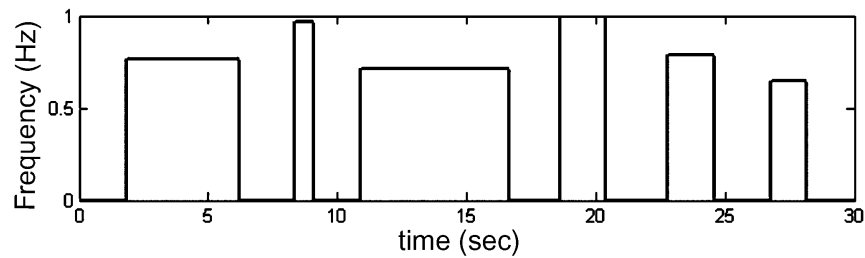

(b)

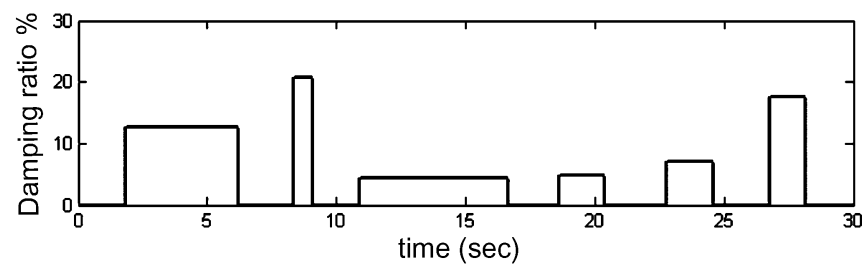

(c)

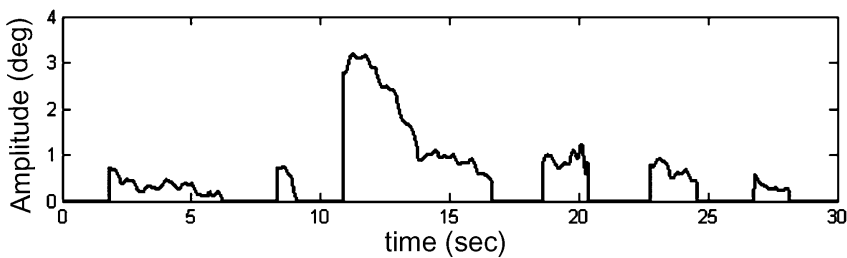

(d)

Fig. 9. Field data and computational results.

\section{CONCLUSIONS}

In this paper, we present a hybrid method which combines four different algorithms including DFT, FIR window filter, LSE, and a prototype algorithm for low-frequency oscillation parameter estimation, each part having its own function. We also use four different kinds of tests to verify this hybrid method, and the computational results are encouraging.

\section{REFERENCES}

[1] A. R. Messina, V. Vittal, D. Ruiz-Vega, and G. Enriquez-Harper, "Interpretation and visualization of wide-area PMU measurements using Hilbert analysis," IEEE Trans. Power Syst., vol. 21, no. 4, pp. 1763-1771, Nov. 2006.

[2] J. Rasmussen and P. Jorgensen, "Synchronized phasor measurements of a power system event in eastern Denmark," IEEE Trans. Power Syst., vol. 21 , no. 1 , pp. 278-284, Feb. 2006.

[3] I. Kamwa, J. Beland, G. Trudel, R. Grondin, C. Lafond, and D. McNabb, "Wide-area monitoring and control at Hydro-Quebec: Past, present and future," in Proc. IEEE Power Eng. Soc. General Meeting, 2006.

[4] S. S. Tsai, Z. Zhong, J. Zuo, and Y. Liu, "Analysis of wide-area frequency measurement of bulk power systems," in Proc. IEEE Power Eng. Soc. General Meeting, 2006.

[5] T. Hashiguchi, M. Yoshimoto, Y. Mitani, O. Saeki, and K. Tsuji, "Analysis of power system dynamics based on multiple synchronized phasor measurements," in Proc. IEEE Power Eng. Soc. General Meeting, 2003.

[6] K. P. Poon and K. C. Lee, "Analysis of transient stability swings in large interconnected power systems by fourier transformation," IEEE Trans. Power Syst., vol. 3, no. 4, pp. 1573-1581, Nov. 1988. 
[7] K. C. Lee and K. P. Poon, "Analysis of power system dynamic oscillations with beat phenomenon by fourier transformation," IEEE Trans. Power Syst., vol. 5, no. 1, pp. 148-153, Feb. 1990.

[8] C. L. Chang, A. S. Liu, and C. T. Huang, "Oscillatory stability analysis using real-time measured data," IEEE Trans. Power Syst., vol. 8, no. 3, pp. 823-829, Aug. 1993.

[9] T. Hashiguchi, M. Yoshimoto, Y. Mitani, O. Saeki, and K. Tsuji, "Analysis of power system dynamics based on multiple synchronized phasor measurements," in Proc. IEEE Power Eng. Soc. General Meeting 2003, Jul. 2003, vol. 2.

[10] P. Korba, M. Larsson, and C. Rehtanz, "Detection of oscillations in power systems using Kalman filtering techniques," in Proc. 2003 IEEE Conf. Control Applications, Jun. 2003, vol. 1, no. 1, pp. 183-188.

[11] J. F. Hauer, "Application of prony analysis to the determination of modal content and equivalent models for measured power system response," IEEE Trans. Power Syst., vol. 6, no. 3, pp. 1062-1068, Aug. 1991.

[12] D. Trudnowski, J. Johnson, and J. Hauer, "Making prony analysis more accurate using multiple signals," IEEE Trans. Power Syst., vol. 14, no. 1, pp. 226-231, Feb. 1999.

[13] D. J. Trundnowski, "Order reduction of large-scale linear oscillatory system models," IEEE Trans. Power Syst., vol. 9, no. 1, pp. 451-458, Feb. 1994.

[14] D. J. Xu, R. M. He, and T. Xu, "A new approach to power system electromechanical oscillation research," Power Systems and Coтmunications Infrastructures for the Future, Sep. 2002.

[15] M. Amono, M. Watanabe, and M. Banjo, "Self-testing and self-tuning of power system stabilizers using Prony analysis," in Proc. IEEE Power Eng. Soc. 1999 Winter Meeting, Feb. 1999, vol. 1, pp. 655-660.

[16] J. Xiao, X. Xie, Y. Han, and J. Wu, "Dynamic tracking of low-frequency oscillations with improved prony method in wide-area measurement system," in Proc. IEEE Power Eng. Soc. General Meeting, 2004, Jun. 2004, pp. 1104-1109.

[17] M. Meunier and F. Brouaye, "Fourier transform, wavelets, Prony analysis: Tools for harmonics and quality of power," in Proc. 8th Int. Conf. Harmonics and Quality of Power 1998, Oct. 1998, vol. 1, pp. 71-76.

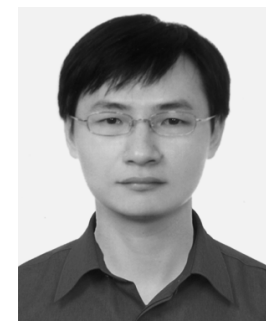

Jun-Zhe Yang (M'00) was born in Tainan, Taiwan, R.O.C., in 1971. He received the B.S. degree in electrical engineering from Tantung University, Taipei, Taiwan, in 1994 and the M.S. and Ph.D. degrees in electrical engineering from Taiwan University, Taipei, in 1997 and 2000, respectively.

After two years in compulsory military service, he joined I-Shou University, Kaoshiung, Taiwan, in 2003, where he is an Assistant Professor of electrical engineering. His research areas are power quality and computer relaying.

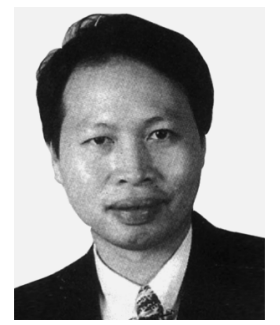

Chih-Wen Liu (S'93-M'96-SM'03) was born in Taiwan, R.O.C., in 1964. He received the B.S. degree in electrical engineering from National Taiwan University, Taipei, Taiwan, in 1987, and the M.S. and $\mathrm{Ph} . \mathrm{D}$. degrees in electrical engineering from Cornell University, Ithaca, NY, in 1992 and 1994, respectively.

Since 1994, he has been with National Taiwan University, where he is a Professor of Electrical Engineering. His main research interests include application of computer technology to power system monitoring, operation, protection, and control.

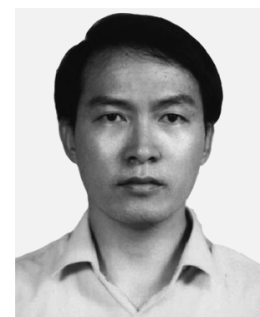

Wen-Giang Wu was born on February 27, 1957, in Taiwan, R.O.C. He received the B.S. degree in computer science from National Cheng Kung University, Tainan, Taiwan, in 1979

Since 1985, he has been with ADX Corporation, Taiwan, where he is currently the Director. His major fields are design and manufacture of power quality recording instruments and synchronized phasor measurement units. His current research interests include real-time power system monitoring on the basis of an $\mathrm{N}$-tiered web server. 